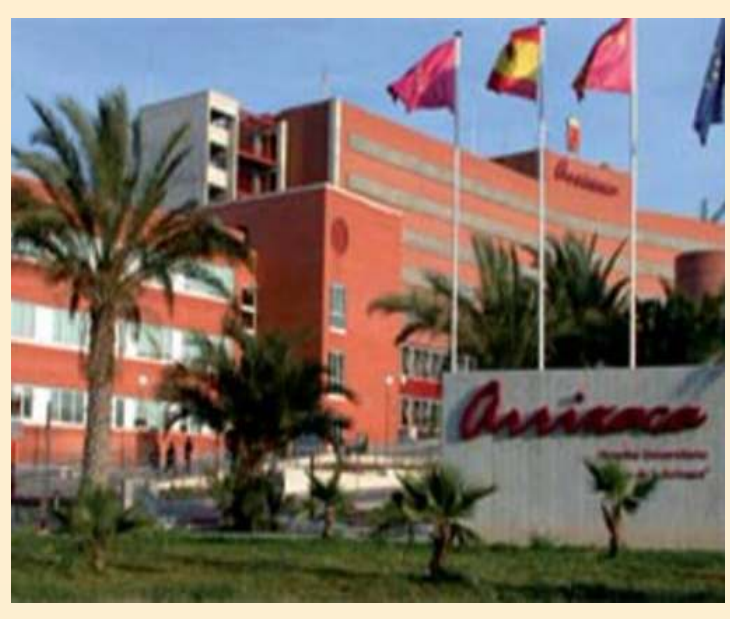

\title{
The infrecuent cerebral anterior artery strokes, special characteristics to take into account. Analysis of a sample.
}

AUTHORS: Vázquez Lorenzo, Julián; Alba Isasi, María Teresa; Palao Rico María; Valero López, Gabriel; Hernández Clares Rocío; Díaz Pérez, José; Baidez Guerrero, Ana Esther; Cabrera Maqueda, José María; Rumí Fuentes, Luna; García Molina, Estefanía; Albert Lacal, Laura.

Servicio de Neurología Hospital Universitario Virgen de la Arrixaca.

\section{BACKGROUND AND PURPOSE}

Cerebral anterior artery strokes (CAA) are very infrecuent and there are few cohorts published. They have special clinical, etiological and prognostic characteristics which are important to be known.

\section{METHODS}

We put forward a descriptive analysis of patients who suffered a stroke in the CAA territory admitted to the Virgen de la Arrixaca Hospital from 2009 until November. We evaluate demographic and clinical characteristics, treatment with thrombolytic therapy and outcomes. We consider good functional outcome Rankin scale $(m R s) \leq 2$. We analyse the relation between neurological deficit at presentation (NIHSS) and treatment received with dependency at 3 months (mRs). We use SPSS 22,0 statistic

\section{RESULTS}

- In the period of our study only 17 strokes involved CAA territory isolated $(0.62 \%)$ from a total population of 2764 strokes. This percentage is similar to the samples published (0.4-3\%).

- 13 of the patients were male $(76,47 \%)$, while only 4 were women. Medium age was 70 .

- We found two bilateral strokes: A1 oclusion in a patient with hypoplasic contralateral CAA. Left internal carotid suboclusion and stenosis $>60 \%$ in right internal carotid with bilateral artery-to-artery emboli.

- Thrombolitic treatment with alteplase was given in $27.8 \%$. The main cause of exclusion was to reach the hospital out of time ( $>4.5$ hours).

- Cardioembolic etiology was the most frecuent (27.8\%) followed by atherothrombotic and cryptogenic.

- $70,6 \%$ had NIHSS $\leq 8$ at stroke onset. $94,1 \%$ had initial Rankin scale $\leq 2$ (good prognosis), while reevaluation three months after stroke reduced the percentage to $35,3 \%$.

- We did not found statistical relation between low NIHSS score and $m R s \leq 2(p \geq 0.05)$. $75 \%$ of patients with unfavorable $m R s$ scale $(\geq 3)$ three months after stroke had not received thrombolitic treatment. We did not find prognosis differences between the group that received thrombolitic therapy and the one that did not received it, although it may be influence by the small sample.

\begin{tabular}{|l|l|l|}
\cline { 2 - 3 } \multicolumn{1}{c|}{} & YES & NO \\
\hline Arterial hypertension & $14(82 \%)$ & $3(8 \%)$ \\
\hline Dyslipemia & $7(41 \%)$ & $10(59 \%)$ \\
\hline Diabetes & $8(47 \%)$ & $9(53 \%)$ \\
\hline Chronic atrial fibrillation & $2(12 \%)$ & $15(88 \%)$ \\
\hline Recent atrial fibrillation & $2(12 \%)$ & $15(88 \%)$ \\
\hline Smoking & $4(24 \%)$ & $13(76 \%)$ \\
\hline AAS & $8(47 \%)$ & $9(53 \%)$ \\
\hline Anticoagulant & $3(18 \%)$ & $14(82 \%)$ \\
\hline
\end{tabular}

\begin{tabular}{|c|c|c|c|}
\cline { 2 - 3 } \multicolumn{1}{c|}{} & $\mathrm{mRs} \geq 3$ & $\mathrm{mRs} \leq 2$ & \multicolumn{1}{c|}{} \\
\hline $\begin{array}{c}\text { Thrombolitic } \\
\text { treatment }\end{array}$ & $3(25 \%)$ & $2(40 \%)$ & $\begin{array}{c}\text { OR 0.5 (0.55-4.6) } \\
\mathrm{p} \leq 0.536\end{array}$ \\
\hline $\begin{array}{c}\text { No thrombolitic } \\
\text { treatment }\end{array}$ & $9(75 \%)$ & $3(60 \%)$ & \\
\hline NIHSS $\leq 8$ & $7(58.3 \%)$ & $5(100 \%)$ & OR 0.583(0.362-0.941) \\
\hline NIHSS 88 & $5(41.7 \%)$ & 0 & $p \leq 0.86$ \\
\hline
\end{tabular}

\section{CONCLUSIONS}

- CAA strokes are quite unusual.

- Low NIHSS scores have no relation with better outcome. It may be due to the lower limb paresis predominantly, which restrain the gait. Neurocognitive impairment may also influence.

- Neurocognitive evaluation should be done in these patients because related sequels may influence the prognosis. Frontal test assesment might be useful.

\section{SOURCES}

Spectrum of anterior cerebral artery territory infarction: clinical and MR findings. E.Kumral et al. EJN 2002,9:615-624;

- Anterior cerebral artery infarction.Stroke mechanism and clinical-imaging study in 100 patients. Suk Y Kang et al. Neurology 70, 2008;

- Clinical and MRI patterns of pericallosal artery infarctions: the significance of supplementary motor area lesiones.Angelica Alonso et al. J.Neurol(2012)259:944-951

Infarction in the territory of the anterior cerebral artery: clinical study of 51 patients. Adria Arboix et al. BMC neurology 2009,9:30. 